\title{
Emotional Responses to Food Pictures according to Caloric Value in Women with an Eating Disorder
}

\section{Running title: Food pictures, Emotions and Eating Disorders}

Caroline Gagnon ${ }^{1}$, Simon Grondin ${ }^{1}$, Marilou Côté ${ }^{1,}$, Marie-Ève Labonté ${ }^{2,}$, Véronique Provencher ${ }^{2,3}$, Carole Ratté ${ }^{4}$, \& Catherine Bégin 1, 2

${ }^{1}$ School of Psychology, Laval University, Quebec, Canada

${ }^{2}$ Institute of Nutrition and Functional Foods, Laval University, Quebec, Canada

${ }^{3}$ School of Nutrition, Laval University, Quebec, Canada

${ }^{4}$ Programme d'Intervention des Troubles des Conduites Alimentaires - Centre Hospitalier Universitaire de Québec, Quebec, Canada

*Corresponding Author: Catherine Bégin, School of Psychology, Laval University, 2325, rue des Bibliothèques, Québec (Québec), Canada, G1V $0 \mathrm{~A} 6$

Received date: August 19, 2021; Accepted date: September 1, 2021; Published date: October 08, 2021

Citation: Gagnon C., Grondin S, Côté M., Marie-È. Labonté, Provencher V., Ratté C., Bégin C. (2021) Emotional responses to food pictures according to caloric value in women with an eating disorder. J. Psychology and Mental Health Care. 5(2): DOI: 10.31579/2637-8892/140

Copyright: (02021 Catherine Bégin, This is an open access article distributed under the Creative Commons Attribution License, which permits unrestricted use, distribution, and reproduction in any medium, provided the original work is properly cited.

\begin{abstract}
Objective: The aim of the current study was to improve the understanding of emotions evoked by food pictures in women with an eating disorder (ED), by distinguishing anorexia nervosa (AN) and bulimia nervosa (BN) diagnoses, while taking into account the caloric content of food and the influence of participants' nutritional knowledge.

Methods: Thirteen AN, 9 BN and 22 healthy controls (HC) women participated in the study. In a laboratory setting, participants first completed self-report questionnaires regarding their affective state. Then, an emotional rating task of food and non-food pictures was performed in order to examine participants' emotional reactions to these pictures, depending on the caloric value of the food depicted and controlling for internal state. Finally, an energy density ranking task of food pictures was completed to investigate participants' nutritional knowledge and its influence on their reactions to food.
\end{abstract}

Results: Compared to HC, ED participants experienced more fear towards food, which was neither due to their internal state nor to their nutritional knowledge. In AN, fear occurred towards all food, whereas in BN, fear was observed for high-calorie products only.

Conclusion: The key role of food-induced fear in ED was highlighted, particularly in AN.

Keywords: affective state; caloric value; eating disorders; emotions; food pictures; nutritional knowledge

\section{List of abbreviations}

AN: Anorexia nervosa;

BMI: Body mass index;

BN: Bulimia nervosa;

ED: Eating disorders;

HC: Healthy controls;
M: Mean;

SD: Standard deviation.

\section{Introduction}

Eating behaviors are known to be influenced by affective states (AguiarBloemer \& Diez-Garcia, 2018; Devenport et al., 2019; Macht \& Simons, 2000; van Strien et al., 2013). Emotions aroused by food are powerful determinants of individual's food choices and intakes (Lowe et al., 2016; 
Martins \& Pliner, 2005; Spence et al., 2016). Indeed, positive emotions evoked by food can drive eating behaviors such as disinhibition or craving, whereas negative emotions can lead to its rejection or avoidance (Macht, 2008). This association between food-induced emotions and eating behaviors seems particularly relevant for individuals suffering from an eating disorder (ED) (for review, see Giel et al., 2011). In fact, ED women present altered information processing mechanisms and erroneous thoughts about food and body weight/shape, which modulate negatively their emotional responses towards food and, thus, their caloric intake (Brooks et al., 2011; Coelho et al., 2014; Eiber et al., 2005; Williamson et al., 1999). Due to their enduring nature, these cognitions and reactions contribute to the maintenance and the worsening of patients' feeding behaviors and their long-term consequences on physical health and psychosocial functioning (Dakanalis et al., 2017; Fairburn et al., 2003; Vitousek \& Brown, 2015). Therefore, in order to better understand ED women's eating behaviors and improve the efficacy of their treatments, it is important to pursue the exploration of emotional responses evoked by food.

In that perspective, Hay and Katsikitis (2014) collected and compared emotional responses generated by food pictures from three groups of women, namely psychiatric controls, ED participants and healthy controls (HC), while adjusting for their initial affective state. This adjustment was important knowing that prevalence of depressive and anxious symptoms is high among individuals suffering from an ED (Aspen et al., 2014; Godart et al., 2015, Morzola et al., 2020 Swinbourne et al., 2012), and considering that such comorbidities can enhance attention allocation to negative stimuli or reduce the experience of positive emotions (Cisler \& Koster, 2010; Dai \& Feng, 2011; Eizenman et al., 2003; Okon-Singer, 2018). Their results revealed that ED participants gave higher negative ratings (i.e., fear, disgust) and lower positive ratings (i.e., happiness/joy) to food pictures than psychiatric participants and HC. Not only did Hay and Katsikitis (2014) confirm what had already been shown in past studies (e.g., Santel et al., 2006, Rodríguez et al., 2007), but they went further by revealing that the differences between groups remained significant despite the statistical control for women's pre-experimental affective state, suggesting that negative food-induced reactions could be a core feature of ED. More recently, Foroughi and colleagues (2018) invited HC and women suffering from anorexia nervosa (AN), atypical AN (i.e., with a body mass index $[\mathrm{BMI}] \geq 18 \mathrm{~kg} / \mathrm{m}^{2}$ ), bulimia nervosa $(\mathrm{BN})$ and binge eating disorder (BED) to rate their level of happiness, fear and disgust prior to and whilst viewing food pictures. Compared with $\mathrm{HC}$, all groups of ED participants felt more fearful and disgusted when viewing food images, even when their pre-experimental mood was controlled for. However, within the four ED groups, no significant differences were detected, suggesting that aversive responses to food could be a transdiagnosis phenomenon. Although interesting, these results contrast with those of other studies suggesting that individuals with BN behaviors can have positive, or at least, less negative reactions than AN individuals when seeing food (Burmester et al., 2021; Drobes et al., 2001; Friederich et al., 2006; Gagnon et al., 2018; Hoefling et al., 2009; Leehr et al., 2016; Racine et al., 2018).

Furthermore, it has been suggested that emotions induced by food pictures in ED women can differ according to their caloric value. By means of semi-structured interviews inviting participants to freely identify their thoughts (i.e., cognitions, emotions) during the visualisation of food pictures, McNamara and colleagues (2008) showed that an impression of negative control over the food ${ }^{1}$-which was predominantly evoked by

\footnotetext{
${ }^{1}$ i.e., the loss of control over food intake and related weight gain or purging behaviors.

2 As far as we know, past literature has focused on patients' subjective perception of fat and caloric contents of food items and
}

high-calorie products-was accompanied by negative emotions (e.g., fear, disgust, guilt), while an impression of positive control over the foodwhich was mostly provoked by low-calorie items-was associated with positive emotions (e.g., happiness, safety).

A study conducted among college women emphasizes the importance of including the caloric value of food. Racine (2018) demonstrated that response towards high or low-calorie food images depended on different dimensions of dietary behaviors. More specifically, real decrease of food intake (i.e., dietary restriction) was associated with reduced pleasure responses towards both high and low-calorie food, while attempts to eat less or cognitive efforts to avoid eating (i.e., cognitive restraint) was associated to greater pleasure ratings for low-calorie food. Taken together, these results suggest that the caloric value of food might influence emotional reactions according to the type of restriction. Then, when examining ED individuals' emotional reactions to food, it seems essential to include caloric value of food. In fact, there may be no difference in emotional rating of food between ED groups when the caloric value is not considered-like in Foroughi et al. (2018)'s experiment-but there may be some differences between $\mathrm{AN}$ and BN women if the energy content of foods is taken into account.

Finally, some studies have already yielded a better knowledge of the nutritional value of food in ED participants, in comparison to healthy women (Beumont et al., 1981; Laessle et al., 1988). As AN and BN individuals are concerned about their body appearance (weight, shape), they are likely interested in nutrition-related issues (American Psychiatric Association [APA], 2013; Robert-McComb et al., 2012). For instance, they can read extensive literature about nutrition to find new strategies to limit their food intake and/or to increase their caloric expenditure (Laessle et al., 1988). Some even consult fitness blogs and pro-ED websites (Mento et al., 2021: Mulveen \& Hepworth, 2006; Rouleau \& von Ranson, 2011; Rodgers et al., 2012). Given these elements, the idea that ED individuals may have better nutritional knowledge than the general population may be still relevant. Knowing that the more caloric the food is perceived, the more intense the negative reaction is likely to be (Gonzalez \& Vitousek, 2004; Houben et al., 2010), it is relevant to believe that ED individuals' nutritional knowledge can influence their emotional reactions to food. However, up until now, no study has examined the effects of objective ED women's nutritional knowledge on their emotional responses to food ${ }^{2}$, and no experiment has investigated for possible differences between $\mathrm{AN}$ and $\mathrm{BN}$ women about their knowledge.

The aim of the present study was to examine ED women's emotional responses to food pictures, and to compare these responses according to their diagnosis, while taking into account both the caloric content of the food items presented and participants' nutritional knowledge. Regarding emotional responses to food, we predicted that women suffering from an ED would present lower positive and higher negative reactions to food pictures than HC. Moreover, we hypothesized that AN participants would show more aversive reactions to food of low and medium energy density than $\mathrm{BN}$ women (APA, 2013). We also predicted that $\mathrm{AN}$ and $\mathrm{BN}$ women would have a better knowledge than $\mathrm{HC}$ about the energy content of the food depicted. Finally, we hypothesized that their knowledge would contribute to explain their unfavorable emotional responses to food, particularly for AN women.

\section{Methods}

meals (e.g., Gonzalez \& Vitousek, 2004; Sunday et al., 1992), not on objective knowledge about food products (i.e., its accuracy). 


\section{Participants}

Twenty-two women suffering from an ED and $22 \mathrm{HC}$ women took part in the study. ED participants were recruited among outpatients of the Programme d'intervention des troubles des conduites alimentaires (PITCA) du Centre Hospitalier Universitaire (CHU) de Québec $(n=12)$, a multidisciplinary hospital unit for the assessment and treatment of ED, and among users of La masion l'Éclaircie services, a community-based organization for individuals presenting ED symptoms in Quebec city $(n=$ $10)$. HC women were recruited among Laval University's community by email advertisements $\left(\mathrm{M}_{\mathrm{age}}=23.41 \pm 5.38\right.$ years; $\mathrm{M}_{\mathrm{BMI}}=21.59 \pm 2.25$ $\mathrm{kg} / \mathrm{m}^{2}$ ). In accordance with the criteria of the Diagnostic and Statistical Manual of Mental Disorders, Fifth Edition (DSM-5; APA, 2013), participants suffering from an ED were divided into two groups: the AN group $\left(n=13 ; \mathrm{M}_{\mathrm{age}}=22.92 \pm 3.99\right.$ years; $\left.\mathrm{M}_{\mathrm{BMI}}=18.91 \pm 1.86 \mathrm{~kg} / \mathrm{m}^{2}\right)$ and the $\mathrm{BN}$ group $\left(n=9 ; \mathrm{M}_{\mathrm{age}}=29.33 \pm 9.68\right.$ years; $\mathrm{M}_{\mathrm{BMI}}=25.96 \pm 5.79$ $\mathrm{kg} / \mathrm{m}^{2}$ ). Participants from the PITCA received their ED diagnosis from a psychiatrist specialized in ED (CR), while women from La Maison l'Éclaircie received theirs from a PhD student in psychology specialized in ED (CG), which was confirmed by an experienced clinical-researcher in the domain of ED (CB).

For the three groups, inclusion criteria were: a) being aged between 18 and 60 years old; b) understanding and speaking French; c) being righthanded; d) having normal or corrected-to-normal vision and audition; e) being free of drug and alcohol abuses for 3 months; $f$ ) presenting neither a psychotic disorder nor a neurological disorder; g) not showing any history of traumatic brain injury within the last 5 years; and h) not being pregnant. Furthermore, women of the HC group should not present: a) personal or family ED history; b) attempts to lose weight in the last month, and c) psychiatric disorders for which medications were prescribed and taken $^{3}$.

\section{Procedure}

Women suffering from an ED were tested in their usual health care setting, whereas $\mathrm{HC}$ were hosted at a research laboratory. All participants gave informed written consent prior to their participation. To reduce the influence of hungriness on tasks' results ${ }^{4}$, all participants were instructed to eat in the 60 minutes preceding their experimental session and were met at fixed hours (i.e. after breakfast, 8:30 am; or after lunch, 1:30 pm). The session lasted around 1.5 hour. First, women had to mention the time and the content of their preceding meal, estimate their level of appetite on a 7-point Likert scale ( $0=$ I am not hungry; $6=$ I am extremely hungry), and fill in questionnaires about their affective state (i.e., BDI-II, STAIYA). Subsequently, they had to complete the emotional rating task of food and non-food pictures. A 10-min break was then taken considering the mental load associated to the first part of the session. Finally, participants performed the energy density ranking task of food pictures. They all received a monetary compensation of $14 \$ C A D$ for taking part in the study. The experiment was approved by the Ethics Committee of the CHU de Quebec (Project 2012-8 12, C11-08-088).

Tasks.

\section{Emotional rating}

${ }^{3}$ At first sight, some of participants' selection criteria might be construed as strict. It must be noted that this study was included in a larger research protocol, which involved cognitive processes evaluation (e.g., time perception) and neuropsychological measures (e.g., attention, working memory, executive functions).
Participants were seated in front of a 16-inch LCD monitor connected to a PC, at a viewing distance of $60 \mathrm{~cm}$. The room was dimly lit. The software E-Prime 2.0 Professional (Psychology Software Tools, Pittsburgh, PA, and Released 2012) was employed to create and administer the task. The food and object pictures were presented in a 700 x 526 pixels colored format, at the center of the screen, on a black font. The stimuli were shown for 5 seconds, one item at a time, in a random order. Not to influence the affective rating of pictures, their specific name or nature (e.g., "garlic pork sausage", "pincers") was not provided. When a food picture was presented, women were instructed to imagine, as vividly as possible, that they were eating the product/meal depicted (i.e., salient or intent-to-eat context; Murray \& Strigo, 2018). When an object picture was displayed, they were asked to imagine themselves as using the item exposed or being in its presence. After each stimulus presentation, participants had to rate the emotions elicited by the item depicted on nine dimensions: valence, arousal, happiness/joy, sadness, anger, disgust, fear, surprise and neutrality. Women were not constrained by a limited amount of time, but they were told to share their immediate and instinctive emotional reactions towards the stimuli. For each picture, the last dimension to evaluate was followed by a short recovery period of 3 seconds, and then the next image to rate was presented. To reduce attentional errors and avoid participant's confusion, the affective dimensions were always surveyed in the same order.

The valence and arousal levels of stimuli were investigated via SelfAssessment Manikin scales (SAM; Bradley \& Lang, 1994; Lang, 1980), which are pictorials tools used for the measurement of a person's affective response to stimuli. The SAM scales were depicted with a 9-point gradation, each character-and the space between two adjacent characterscorresponded to an ascending intensity level ranging from 1 (negative valence or low arousal) to 9 (positive valence or high arousal). For the remaining seven affective dimensions (i.e., happiness/joy, sadness, anger, disgust, fear, surprise and neutrality), Likert scales ranging from 1 (none) to 9 (very strongly), were used to assess emotions and the degree to which they experienced it. Ratings were collected by a numeric keypad. Before starting the task, in the presence of the experimenter (CG), participants made a practice trial with an object picture (i.e., a clock).

\section{Energy density ranking}

With all the stimuli in front of them, participants were instructed to rank the food from the lowest energy-dense (least kcal/g) to the highest energydense (most $\mathrm{kcal} / \mathrm{g}$ ), according to their knowledge. A dry-erase marker and a table-napkin were given to participants, so they could write on the pictures and correct their classification easily. When they were confident about their ranking, participants were invited to transcribe it on an answer form.

\section{Measures}

\section{Screening of ED symptomatology.}

Module $\mathrm{H}$ of the Structured Clinical Interview for DSM-IV-TR Axis I Disorders - Research version, Patient Edition (SCID-I/P; First et al., 2002), adapted for the DSM-5 criteria (APA, 2013), was used to diagnose ED. The SCID-I/P has an interjudge reliability of .70-1.0 and a test-retest reliability of .82-.90 for both clinical and community samples (First et al., 2002; Pike et al., 1995; Segal et al., 1994; Williams et al., 1992).

Therefore, it was essential to limit, as far as possible, the influence of factors that could alter women's cerebral functioning.

${ }^{4}$ Appetite modulates individuals' affective (valence, arousal), cerebral (activation, orientation of attention) and psychophysiological (heart rate, startle blink reflex) responses to food cues (e.g., Piech et al., 2010; Hoefling et al., 2009; Stockburger et al., 2009; Stoeckel et al, 2007; Uher et al., 2006). 
Diagnostic questions of the Eating Disorder Examination- Questionnaire (EDE-Q 6.0; Fairburn \& Beglin, 2008) were used to validate the absence of significant ED manifestations in participants of the HC group. The instrument, requiring answers on 7-point Likert scales ranging from 0 (no day, not at all) to 6 (every day, markedly), addresses the core attitudinal features and behaviors of ED pathology over the past 28 days. To be included in the study as HC, women should not present fasting (score = 0 ) or important restrained eating to lose weight (score $<3$ ), nor episode of binge eating accompanied by a loss of control and usage of inappropriate behaviors to prevent weight gain (score $=0$; ref. criteria used by Tokley \& Kemps, 2007; Kemps \& Wilsdon, 2010). Additionally, HC participants had to be satisfied with their weight and body (dissatisfaction score < 3), and these elements could not affect significantly their self-perception (score < 3). The EDE-Q has a satisfactory internal consistency ( $\alpha=.78-.93$ for community sample; Luce \& Crowther, 1999; Mond et al., 2004a; Peterson et al., 2007) and an acceptable test-retest reliability: $(r=.81-.94$ for 2 weeks, $r=.57-77$ for about one year; Luce \& Crowther, 1999; Mond et al., 2004b).

\section{Assessment of participants' comorbid affective state.}

Depressive symptoms of participants were assessed by the Beck Depression Inventory II (BDI-II; Beck et al., 1996), a self-report questionnaire consisting of 21 items answered on a 4-point Likert scale ranging from 0 to 3 (example item: Crying; $0=$ "I don't cry any more than I used to"; 1 = "I cry more than I used to"; 2 = "I cry over every little thing"; 3 = "I feel like crying, but I can't"). The BDI-II total score assesses respondents' depressive mood in the past two weeks, with higher scores indicating more severe depressive symptoms. The questionnaire has a high internal consistency $(\alpha=.90)$ and a good test-retest stability $(r=.73$ .96; for review, see Wang \& Gorenstein, 2013).

Anxiety symptoms of participants were assessed with the Part A of the State-Trait Anxiety Inventory-Y Form (STAI-YA, Spielberger, 1983). The STAI-YA measures State-Anxiety, which can be defined as feelings of apprehension, tension, nervousness and worry induced temporarily by situations perceived as dangerous. Twenty items, assessed on 4-point Likert scales, evaluate how respondents feel "right now" or felt "in a recent past" (example item: "I worry too much over something that really doesn't matter") A global score was computed by summing all items. Scores were then converted into an age-dependent T-score. The internal consistency of the whole inventory (Parts A and B) ranges from .86 to .95 and its test-retest reliability ranges from .31 to .86 (intervals fluctuating from 1 hour to 104 days; Gauthier \& Bouchard, 1993; Spielberger, 1983).

\section{Stimuli.}

\section{Emotional rating task}

Out of the 58 images displayed, 46 were food pictures and 12 were nonfood pictures, i.e. object pictures (including one object picture used for a practice trial). Food pictures $(n=30)$ were chosen from the Institute of Nutrition and Functional Foods' web-based food frequency questionnaire (Web-FFQ; Labonté et al., 2012), an online dietary assessment tool developed for qualifying and quantifying French Canadians' dietary intakes using digital colored photographs of various food items and meals. The Web-FFQ was used to avoid potential limits and biases due to stimuli (Piqueras-Fiszman \& Spence, 2014; Spence et al., 2016), because: a) its pictures are standardized in terms of viewing point, brightness, shading, background and cropping; b) its food products are presented with the same dinnerware and utensils, and 3) different portion sizes of food items and meals are available.

To maximise the diversity and the representativeness of food stimuli showed to women, some criteria guided the selection of the photographs: 1) the four main categories of Canada's Food Guide (i.e., Vegetables and fruits, Grain products, Milk and alternatives, Meat and alternatives; Health Canada, 2011) and its "Other Foods" category (i.e., products that cannot be classified in the main categories, such as candies, chips, and pastries) had to be represented; 2) a reasonably comparable number of unprocessed and minimally-processed foods vs. moderately- and highlyprocessed foodstuffs had to be illustrated; 3 ) all food products had to be displayed on the same dinner plate, surrounded by the same fork and knife (i.e., beverages dispensed in a glass or a cup, and foods presented in a bowl were excluded); 4) the orientation and the localisation of the plate's content had to be similar from one picture to another (i.e., pointing towards participants and in the middle of the plate, centered; Michel et al., 2015a,b; Rowley \& Spence, 2018); 5) the plate's area covered by foods had to be equivalent between stimuli minimally (Herzog et al., 2017; Kissileff et al., 2016); 6), the general nature of the food items and meals had to be easily identified or recognized (e.g., meat, cheese, pie); 7) based on past researches, foods for which positive and negative emotions could be evoked in ED and/or HC women had to be equally illustrated (e.g., strawberries, piece of cake, shellfish; Blechert et al., 2014; Charbonnier et al., 2016; Foroni et al., 2013; McNamara et al., 2008; Miccoli et al., 2016); and 8) low (< $1.5 \mathrm{kcal} / \mathrm{g})$, medium (1.5-4.0 $\mathrm{kcal} / \mathrm{g}$ ) and high (> $4.0 \mathrm{kcal} / \mathrm{g}$ ) energy density foods had to be shown across the collection of images selected (Rolls \& Barnett, 2000). Figure 1 shows examples of food stimuli used in the experiment's tasks.
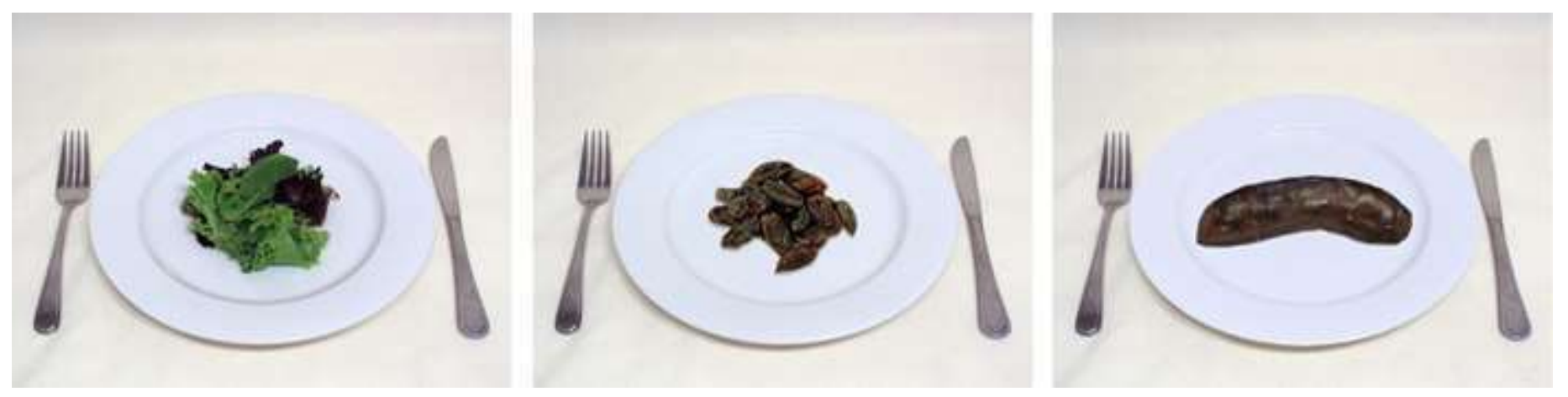

Figure 1. Examples of food pictures used in the emotional rating task.

The object pictures were extracted from the International Affective Picture System (IAPS; Lang et al., 2008), a widely exploited database of images for the study of emotion. As for food stimuli, the selection of object pictures relied on specific criteria: 1) the objects had to be of common use or well-known; 2) they could not refer to foods, beverages or eating; 3 ) as much as possible, their size had to be similar from one picture to another, and to be equivalent to the portion size of food pictures previously chosen; 4) their background color had to be close to the one of 
food images (i.e., light gray beige). Moreover, according to the affective normative data of the IAPS (values on 9-point scales, ranging from 1 [negative or low] to 9 [positive or high]), the images must had: 1) a value between 4 and 6 on the Valence scale of Lang et al. (2008)'s data; 2) a value $<5$ on the Emotion scales (i.e., happiness/joy, surprise, sadness, anger, disgust and fear) of Libkuman et al. (2007)'s ratings; and 3) a low Arousal value $(<5)$ as reported in Lang et al. (2008)'s material. In accordance with all these guidelines, the following IAPS stimuli were chosen: \#6150, 7003, 7016, 7018, 7043, 7053, 7056, 7059, 7090, 7175, 7185 , and 7211 . When required, the visual parameters of these images were edited to be as comparable as possible to those of food pictures.

\section{Energy density ranking task}

From the 46 food pictures used in the emotional rating task, 25 were picked up as stimuli for the energy density ranking task, which was designed to assess participants' nutritional knowledge. The food pictures selected represented food items and meals easily found at local supermarkets. The food images were printed in a $8 \times 6 \mathrm{~cm}$ colored format, on a US legal-size $(21.6$ x $35.6 \mathrm{~cm})$ white paper. The stimuli were disposed in two columns of five pictures, totalizing three pages. The pages were laminated to facilitate their manipulation. The name, nature/flavor and cooking mode of the foods (e.g., "long-grain white rice, cooked") were labelled under each stimulus.

\section{Statistical analyses.}

All statistical analyses were carried out with SPSS 24.0 for Windows (IBM Corporation, Released 2016). All study variables were inspected for outliers and normality, and appropriate transformations were applied when needed. For all analyses, the alpha level, which was set to .05 , was Bonferroni-corrected for multiple testing when required (i.e., decomposition of main effects and post-hoc comparisons). Univariate analyses of variance (ANOVAs) with post-hoc Tukey tests were performed to examine mean differences among $\mathrm{AN}, \mathrm{BN}$ and $\mathrm{HC}$ groups on BMI, hungriness and scores on affective state (i.e., BDI-II, STAI-YA). Due to its fixed nature and its non-normal distribution, the mean age of groups was contrasted with non-parametric analyses, i.e. a Kruskal-Wallis test followed by Mann-Whitney U tests.

\section{Emotional rating task}

Participants' ratings of food and object pictures were investigated according to the intensity of their emotional responses. For object pictures, a mean score including all object pictures pooled together was calculated for each emotional dimension and each group of participants surveyed (e.g., "Objects-Valence intensity-AN"). For food pictures, to examine whether women's responses differed according to the energy density of the food displayed, the food stimuli were sorted in three classes, namely low $(<1.5 \mathrm{kcal} / \mathrm{g})$, medium $(1.5-4.0 \mathrm{kcal} / \mathrm{g})$ and high $(>4.0$ $\mathrm{kcal} / \mathrm{g}$ ) energy density products (Rolls \& Barnett, 2000). For each of these classes, each emotional dimension and each group of participants studied, an intensity mean score was computed (e.g., "Low energy densityValence intensity-AN").

In order to determine which variables to include in the group comparison analysis, Pearson correlations were run on the entire sample with emotional mean scores. Because valence, anger and sadness responses were strongly correlated together and with other emotional dimensions $(r$ $=.68$ to .96), they were excluded from the analyses for the sake of parsimony. Surprise ratings were also excluded due to their lower relevance for the present study and their conceptual complexity (for review, see Mellers et al., 2013; Reisenzein et al., 2017). The remaining dimensions of arousal, happiness/joy, disgust, fear and neutrality were compared between $\mathrm{AN}, \mathrm{BN}$ and $\mathrm{HC}$ groups using multivariate analyses of variance (MANOVAs). The decomposition of MANOVAs' main effect (i.e., the multivariate one) was achieved with ANOVAs, then Tukey tests were used for post-hoc comparisons.

To test associations between women's emotional responses and their comorbid affective state, correlation analyses were performed with reactions towards pictures and the STAI-YA scores. When variables were significantly related, the severity of anxiety symptoms was controlled for in the MANOVAs and ANOVAs. Because the STAI-YA and the BDI-II scores were strongly correlated $(r=.86, p<.001)$, only the former was used as a covariate in the analyses. Finally, as the hunger level of women was not correlated with their emotional responses towards food and object pictures (all $p \mathrm{~s}>.05$ ), this variable was not entered as a covariate in the analyses concerning the stimuli-induced reactions.

\section{Energy density ranking task}

Participant's performance on the energy density ranking task was assessed by means of a precision index, which was calculated by adding up the gap, in absolute value, between each food product's real rank and the rank assigned. Thus, the higher the precision index was, the less accurate was women's nutritional knowledge about the energy density of the items displayed. According to their real energy density value, the 25 food products were sorted out as low, medium or high-energy foods, and a precision index was computed for each class, for each participant. To explore the differences between groups on these three classes, a MANOVA was run. The anxious comorbid state was not controlled for in the analyses, because no association was found between participants' precision indexes and their scores on the STAI-YA. For the same reason, the hunger level of women was not entered as a covariate in the analyses.

Finally, to verify if participants' emotional responses to food pictures were explained by their nutritional knowledge, emotional ratings assigned to the 25 food products submitted in the ranking task were retrieved from the rating task's results, and a mean score was calculated for each of the five affective dimensions investigated (i.e., arousal, happiness/joy, disgust, fear, and neutrality). Three multivariate multiple regression analyses (i.e., one per group) were then run to test whether the emotional ratings of food pictures (i.e., the intensity of reactions associated to them, on each dimension) would be predicted by the ranking task's global precision index.

\section{Results}

\section{Group comparisons for age, BMI, hunger and comorbid affective state.}

There were no significant differences between $\mathrm{AN}, \mathrm{BN}$ and $\mathrm{HC}$ women for age, $H(2, N=44)=4.49, p=.106$, and for the level of hungriness preceding the experimental session, $F(2,41)=1.25, p=.296$. However, the groups differed on BMI, $F(2,41)=16.78, p<.001, \eta_{\mathrm{p}}^{2}=.45$, BDI-II scores, $F(2,41)=22.89, p<.001, \eta^{2}{ }_{p}=.53$, and STAI-YA scores, $F(2$, $41)=29.48, p<.001, \eta_{\mathrm{p}}^{2}=.59$. More precisely, AN participants had a lower mean BMI than BN $(p<.001)$ and HC women $(p=.003)$, and $\mathrm{BN}$ participants had a higher mean BMI than $\mathrm{HC}(p=.007)$. For the comorbid affective state, $\mathrm{AN}$ and $\mathrm{BN}$ women showed more depressive $\left(M_{\mathrm{AN}}=22.15\right.$ $\left.\pm 16.03 ; M_{\mathrm{BN}}=16.67 \pm 14.85 ; p<.001\right)$ and anxiety symptoms $\left(M_{\mathrm{AN}}=\right.$ $\left.56.38 \pm 11.96 ; M_{\mathrm{BN}}=58.22 \pm 14.59 ; p<.001\right)$ than $\mathrm{HC}$ did $\left(M_{\mathrm{BDI}-\mathrm{II}}=2.64\right.$ $\left.\pm 2.90 ; M_{\text {STAI }- \text { YA }}=38.95 \pm 5.40\right)$.

\section{Emotional rating task.}

\section{Object pictures.}

For all object pictures, results showed a non-significant multivariate effect of Group ( $p=.560$, see Table 1$)$. Hence, no further analyses were achieved to examine the emotional dimensions. 


\begin{tabular}{|c|c|c|c|c|c|c|c|}
\hline & AN & $\mathrm{BN}$ & $\mathrm{HC}$ & & & & \\
\hline Variables & $M(S D)$ & $M(S D)$ & $M(S D)$ & $\lambda, V$ & $d f$ & $F$ & $\eta_{\mathrm{p}}^{2}$ \\
\hline \multicolumn{4}{|l|}{ Object pictures } & .80 & 10,74 & 0.88 & .11 \\
\hline Arousal & $2.69(1.70)$ & $3.41(1.34)$ & $3.54(1.55)$ & & & & \\
\hline Happiness/joy & $1.66(0.42)$ & $1.80(0.69)$ & $1.69(0.73)$ & & & & \\
\hline Disgust & $1.24(0.53)$ & $1.16(0.17)$ & $1.18(0.34)$ & & & & \\
\hline Fear & $1.50(0.72)$ & $1.48(0.57)$ & $1.39(0.54)$ & & & & \\
\hline Neutrality & $7.29(1.39)$ & $6.38(0.80)$ & $6.45(2.10)$ & & & & \\
\hline
\end{tabular}

Note. $\mathrm{AN}=$ Anorexia nervosa $\mathrm{BN}=$ Bulimia nervosa $\mathrm{HC}=$ Healthy controls; $M=$ Mean; $S D=$ Standard deviation.

\section{Table 1: Mean (and SD) intensity of reactions towards object pictures, for each group.}

\section{Food pictures.}

The MANOVA performed with low energy density food' responses showed a significant multivariate effect of Group $(p<.001$; see Table 2$)$. ANOVAs $(\alpha=.01)$ demonstrated a significant effect of Fear $(p<.001$, $\eta_{p}^{2}=.63$ ). According to post-hoc tests, $\mathrm{AN}$ and $\mathrm{BN}$ women judged low energy density food as more fearful than $\mathrm{HC}$ participants did $(p<.001, p$ $=.003)$. The AN group also reported higher ratings of fear than the $\mathrm{BN}$ group $(p=.004)$. When controlling for anxiety symptoms $(\alpha=.01)$, the Fear effect remained significant $\left(p<.001, \eta_{p}{ }^{2}=.40\right)$, but the difference between $\mathrm{BN}$ and $\mathrm{HC}$ participants on that dimension was no longer statistically significant $(p=.999)$.

\begin{tabular}{|c|c|c|c|c|c|c|c|c|c|c|c|}
\hline & AN & $\mathrm{BN}$ & $\mathrm{HC}$ & & & & & & \multicolumn{3}{|c|}{ Controlling for STAI-YA } \\
\hline Variables & $M(S D)$ & $M(S D)$ & $M(S D)$ & $\lambda, V$ & $d f$ & $F$ & $\eta_{\mathrm{p}}^{2}$ & Post-hoc & $F$ & $\eta_{\mathrm{p}}{ }^{2}$ & Post-hoc \\
\hline \multicolumn{4}{|c|}{ Low energy density foods } & .26 & 10,74 & $7.10 * *$ & .49 & & $3.93 * *$ & .35 & \\
\hline Arousal & $\begin{array}{l}5.15 \\
(1.17)\end{array}$ & $\begin{array}{l}5.14 \\
(1.41)\end{array}$ & $\begin{array}{l}5.15 \\
(1.05)\end{array}$ & & 2,41 & $<.001$ & $<.001$ & - & 2.84 & .12 & - \\
\hline Happiness/joy & $\begin{array}{l}3.50 \\
(0.95)\end{array}$ & $\begin{array}{l}3.26 \\
(1.14) \\
\end{array}$ & $\begin{array}{l}3.44 \\
(0.89)\end{array}$ & & 2,41 & 0.17 & .01 & - & 0.16 & .01 & - \\
\hline Disgust & $\begin{array}{l}3.92 \\
(1.14)\end{array}$ & $\begin{array}{l}3.39 \\
(1.08) \\
\end{array}$ & $\begin{array}{l}3.10 \\
(1.02)\end{array}$ & & 2,41 & 2.40 & .11 & - & 0.97 & .05 & - \\
\hline Fear & $\begin{array}{l}4.30 \\
(1.68)\end{array}$ & $\begin{array}{l}2.48 \\
(1.57)\end{array}$ & $\begin{array}{l}1.19 \\
(0.37)\end{array}$ & & 2,41 & $35.12 * *$ & .63 & $1,2>3 ; 1>2$ & $13.24 * *$ & .40 & $1>2,3$ \\
\hline Neutrality & $\begin{array}{l}3.22 \\
(1.39) \\
\end{array}$ & $\begin{array}{l}3.40 \\
(1.80) \\
\end{array}$ & $\begin{array}{l}3.45 \\
(1.13) \\
\end{array}$ & & 2,41 & 0.12 & .01 & - & 0.81 & .04 & - \\
\hline \multicolumn{4}{|c|}{ Medium energy density foods } & .84 & 10,76 & $5.51 * *$ & .42 & & $3.79 * *$ & .34 & \\
\hline Arousal & $\begin{array}{l}6.16 \\
(1.06)\end{array}$ & $\begin{array}{l}5.35 \\
(1.45)\end{array}$ & $\begin{array}{l}5.53 \\
(1.11)\end{array}$ & & 2,41 & 2.45 & .11 & - & 2.01 & .09 & - \\
\hline Happiness/joy & $\begin{array}{l}2.64 \\
(0.85)\end{array}$ & $\begin{array}{l}2.69 \\
(0.73)\end{array}$ & $\begin{array}{l}3.93 \\
(1.24)\end{array}$ & & 2,41 & $8.09^{*}$ & .28 & $1,2<3$ & 2.23 & .10 & - \\
\hline Disgust & $\begin{array}{l}5.03 \\
(1.64)\end{array}$ & $\begin{array}{l}4.15 \\
(1.46)\end{array}$ & $\begin{array}{l}2.88 \\
(1.10)\end{array}$ & & 2,41 & $10.77 * *$ & .34 & $1>3$ & 1.94 & .09 & - \\
\hline Fear & $\begin{array}{l}6.34 \\
(1.64)\end{array}$ & $\begin{array}{l}3.86 \\
(2.46)\end{array}$ & $\begin{array}{l}1.13 \\
(0.30)\end{array}$ & & 2.41 & $56.07 * *$ & .73 & $1,2>3 ; 1>2$ & $21.57^{* * *}$ & .52 & $1>2,3$ \\
\hline Neutrality & $\begin{array}{l}2.59 \\
(1.14) \\
\end{array}$ & $\begin{array}{l}3.18 \\
(1.45) \\
\end{array}$ & $\begin{array}{l}3.03 \\
(1.44) \\
\end{array}$ & & 2,41 & 0.58 & .03 & - & 0.73 & .04 & - \\
\hline \multicolumn{4}{|c|}{ High energy density foods } & .97 & 10,76 & $7.13 * *$ & .48 & & $4.51 * *$ & .38 & \\
\hline Arousal & $\begin{array}{l}6.87 \\
(1.02)\end{array}$ & $\begin{array}{l}5.31 \\
(1.59)\end{array}$ & $\begin{array}{l}6.11 \\
(1.33)\end{array}$ & & 2,41 & 3.84 & .16 & - & 3.70 & .16 & - \\
\hline Happiness/joy & $\begin{array}{l}2.98 \\
(1.22) \\
\end{array}$ & $\begin{array}{l}2.81 \\
(1.08) \\
\end{array}$ & $\begin{array}{l}5.19 \\
(1.47) \\
\end{array}$ & & 2,41 & $16.22 * *$ & .44 & $1,2<3$ & 3.98 & .17 & - \\
\hline Disgust & $\begin{array}{l}4.77 \\
(2.11)\end{array}$ & $\begin{array}{l}3.65 \\
(1.91)\end{array}$ & $\begin{array}{l}1.94 \\
(0.93)\end{array}$ & & 2,41 & $14.34 * *$ & .41 & $1,2>3$ & 2.08 & .09 & - \\
\hline Fear & $\begin{array}{l}7.15 \\
(1.20)\end{array}$ & $\begin{array}{l}4.60 \\
(2.79)\end{array}$ & $\begin{array}{l}1.06 \\
(0.19)\end{array}$ & & 2.41 & $80.74 * *$ & .80 & $1,2>3 ; 1>2$ & $30.84^{* * *}$ & .61 & $1,2>3 ; 1>2$ \\
\hline Neutrality & $\begin{array}{l}2.16 \\
(1.00)\end{array}$ & $\begin{array}{l}2.85 \\
(1.40)\end{array}$ & $\begin{array}{l}2.91 \\
(1.54)\end{array}$ & & 2,41 & 0.94 & .04 & - & 0.60 & .03 & - \\
\hline
\end{tabular}

Note $1 . \mathrm{AN}=$ Anorexia nervosa $\mathrm{BN}=$ Bulimia nervosa $; \mathrm{HC}=$ Healthy controls $;=$ Mean; $S D=$ Standard deviation.

Note 2. For post-hoc comparisons: $1=\mathrm{AN} ; 2=\mathrm{BN} ; 3=\mathrm{HC}$. 
For medium energy density food, the results also indicated a multivariate effect of Group $(p<.001)$, then ANOVAs $(\alpha=.01)$ showed significant effects for Happiness/Joy $(p=.001)$, Disgust $(p<.001)$ and Fear $(p<$ .001 ) dimensions. The size of the effects found were high, particularly for the Fear dimension $\left(\eta_{p}^{2}=.73\right)$. Post-hoc comparisons showed that the AN and $\mathrm{BN}$ groups had lower happiness/joy responses towards medium energy density foods than the $\mathrm{HC}$ one $(p=.003, p=.014)$. Furthermore, $\mathrm{AN}$ women had greater disgust responses than $\mathrm{HC}(p<.001)$. For fear reactions, $\mathrm{AN}$ participants gave higher ratings than $\mathrm{BN}(p=.001)$ and $\mathrm{HC}$ $(p<.001)$, and $\mathrm{BN}$ participants gave higher ratings than $\mathrm{HC}$ women did $(p<.001)$. When controlling for anxiety symptoms $(\alpha=.01)$, the previously significant Happiness/Joy $(p=.121)$ and Disgust effects were no longer significant $(p=.157)$, but group differences remained on Fear $\left(p<.001, \eta_{p}{ }^{2}=.52\right)$, except the difference between the $\mathrm{BN}$ and $\mathrm{HC}$ groups which was no longer significant $(p=.450)$.

The MANOVA conducted with high energy density food yielded a multivariate effect of Group $(p<.001)$, and consecutive ANOVAs $(\alpha=$ $.01)$ demonstrated significant univariate effects of Happiness/Joy $(p<$ $.001)$, Disgust $(p<.001)$ and Fear $(p<.001)$. Once again, the size of the effects found were high, particularly for the Fear dimension $\left(\eta_{p}^{2}=.80\right)$. Post-hoc comparisons showed that $\mathrm{AN}$ and $\mathrm{BN}$ groups had lower happiness/joy responses $(p<.001, p<.001)$, higher disgust responses $(p$ $<.001, p=.011)$ and greater fear reactions $(p<.001, p<.001)$ to high energy density food than the HC group. In addition, AN women gave higher ratings of fear than $\mathrm{BN}$ participants did $(\mathrm{p}<.001)$. When controlling for anxiety symptoms $(\alpha=.01)$, the Happiness/Joy $(p=.027)$ and the Disgust $(p=.139)$ effects were no longer significant, but this time, the difference between $\mathrm{BN}$ and $\mathrm{HC}$ women on the Fear dimension remained significant $(p=.013)$. The effect size of Fear also remained high, $\eta_{p}^{2}=.61$.

\section{Energy density ranking task}

\section{Nutritional knowledge}

The MANOVA ran with the precision indexes related to food's classes of energy density did not show a significant multivariate effect of Group, indicating that $\mathrm{AN}, \mathrm{BN}$ and $\mathrm{HC}$ women had a comparable nutritional knowledge, $\lambda=.91, F(6,78)=.61, p=.719, \eta_{\mathrm{p}}^{2}=.05($ see Table 3$)$.

\begin{tabular}{|l|l|l|l|l|l|l|}
\hline & $\mathrm{AN}$ & $\mathrm{BN}$ & $\mathrm{HC}$ & & & \\
\hline Variables & $M(S D)$ & $M(S D)$ & $M(S D)$ & $\lambda$ & $d f$ & $F$ \\
\hline Performance according to food products' energy density class & & $\mathbf{. 9 1}$ & $\mathbf{6 , 7 8}$ & $\mathbf{0 . 6 1}$ & $\mathbf{. 0 5}$ \\
\hline Low $^{\mathbf{a}}$ & $16.46(5.46)$ & $16.00(7.07)$ & $15.64(4.95)$ & & & \\
\hline Medium $^{\mathbf{b}}$ & $79.39(7.59)$ & $78.11(11.13)$ & $80.64(8.14)$ & & & \\
\hline High $^{\mathbf{c}}$ & $28.92(6.89)$ & $31.00(5.29)$ & $28.64(6.46)$ & & & \\
\hline
\end{tabular}

Note . Precision index = Sum of the gap, in absolute value, between each food products real rank and the rank assigned by a participant; AN = Anorexia nervosa; $\mathrm{BN}=$ Bulimia nervosa; $\mathrm{HC}=$ Healthy controls; $M=$ Mean; $S D=$ Standard deviation.

a 7 stimuli involved. ${ }^{\mathrm{b}} 13$ stimuli involved. ${ }^{\mathrm{c}} 5$ stimuli involved. Table 3: Mean (and SD) precision index on the energy density ranking task, for each group.

\section{Influence of nutritional knowledge on emotional responses to food pictures.}

The results of the multiple regression analysis $(\alpha=.01)$ conducted for each group (i.e., AN, BN, and $\mathrm{HC}$ ) showed that women's nutritional knowledge about food products did not predict their arousal, happiness/joy, disgust, fear, and neutrality reactions towards them (all $p \mathrm{~s}$ $>$.05). In other words, the intensity of participants' reactions to food from the emotional rating task was not explained by their objective nutritional knowledge.

\section{Discussion}

The objective of the present study was to examine ED women's emotional responses to food pictures, and to compare these responses according to their diagnosis, while taking into account both the caloric content of the food items presented and participants' nutritional knowledge. For this purpose, ED and $\mathrm{HC}$ women participated in an emotional rating task of food and object pictures, and an energy density ranking task of food items/meals. When appropriate, we controlled for the internal state of participants, that is their level of hungriness and their comorbid affective state, in order to reduce the effects of non-specific factors in the interpretation of results.

\section{Emotional responses towards food and object pictures}

Participants' emotional responses according to food's classes of energy density showed that when medium and high energy food were presented, groups clearly differed on the happiness/joy, disgust and fear dimensions, but when low energy items were displayed, only the fear dimension differed across groups. For AN and BN participants, higher fear reactions were observed compared to $\mathrm{HC}$ for all food pictures presented, regardless of their caloric value. AN women also experienced higher fear reactions than $\mathrm{BN}$ participants. In addition, the results showed that while AN participants judged medium and high energy density food as more disgusting than $\mathrm{HC}$ did, $\mathrm{BN}$ women's disgust responses only exceeded those of $\mathrm{HC}$ for high energy food. Therefore, it seems that the caloric value of food necessary to generate disgust has to be higher with $\mathrm{BN}$ than with AN women. These findings are in line with those of Santel et al. (2006) and Rodriguez et al. (2007), which demonstrated respectively that $\mathrm{AN}$ and $\mathrm{BN}$ participants assessed images of high energy food as less pleasant than HC. They are also in accordance with the conclusions of Harvey et al. (2002) and those of Griffiths and Troop (2006), stating that women with high risk for ED rated images of high-density food as more disgusting than women at low risk. However, our findings extend those of previous research by distinguishing emotional responses of ED women to food based on their caloric value. They also support and extend the work of Racine (2018) conducted among college women, which revealed that an effective restriction (as seen in AN participants) is correlated with lower pleasure towards both high and low energy food, while temporary or infructuous attempts to eat less (as seen in BN participants) are associated to greater pleasure for low energy food. Such information is interesting for the development of therapeutic strategies targeting more efficiently patients' cognitions and behaviors.

Regarding the analysis of object pictures, results showed that the three groups of participants did not differ in terms of intensity of their emotional ratings. The inclusion of non-food pictures allowed to verify if participants' reactions were oriented exclusively towards food products or were explained rather by a global limited capacity to resent pleasure and/or a higher disgust sensitivity (see Aharoni \& Hertz, 2012; Boehm et al., 2018; Deborde et al., 2006). The current results suggest that AN and BN participants' negative reactions were oriented exclusively towards food. This finding supports the work of Davey et al. (1998) and Troop 
and al. (2002), which denoted that ED participants exhibit a higher disgust sensitivity for food and body-related stimuli, but not for other items. Consequently, it also refuted the hypotheses raised by some authors about the presence, in ED women, of a global lower disgust tolerance and a reduced general capacity to experience pleasure (Aharoni \& Hertez, 2012; Boehm et al., 2018; Deborde et al., 2006).

\section{Influence of participants' internal state}

The current study also aimed to control for women's internal state. The methodological control we exerted for participants' hungriness, i.e., instructions to eat 60 minutes prior to the experiment and meeting at fixed times of the day, was shown efficient because women's appetite level before completing the tasks was low and did not differ between groups. Besides, the statistical control we applied for participants' anxious state (which were highly correlated with their depressive symptoms) in the analysis of their emotional reactions towards food pictures modified the pattern of the results. Specifically, all group effects for happiness/joy and disgust responses that were demonstrated were no longer significant when controlling for anxious state. In other words, it seems that ED participants' lower happiness/joy and higher disgust reactions towards food pictures were better explained by their anxiety state prior to the experiment. These results are consistent with those of Davey and Chapman (2009), which showed that controlling for anxiety overrides the association between disgust and ED-like symptoms. Accordingly, they postulated that the presence of anxious manifestations in individuals at risk of ED could amplify their experience of distress and negative emotions (e.g., disgust, displeasure) for sensitive stimuli. Building on these findings, anxiety state could have influenced their evaluation of food pictures by contributing to explain their aversive reactions towards them (e.g., less happiness/joy, more disgust).

Furthermore, regarding the fear effect, although it remained significant when controlling for anxiety state, the group differences observed between $\mathrm{BN}$ and $\mathrm{HC}$ women were no longer significant, except for the high-energy food category. These results contrast with those of Hay and Katsikitis (2014), which showed that ED women gave lower happiness and higher fear and disgust responses to food images than $\mathrm{HC}$, even when their baseline level for these three emotions were controlled for. Nonetheless, the fact that the differences between BN and $\mathrm{HC}$ women were no longer significant when controlling for anxiety state on fear ratings for low and medium energy food, but remained significant for high energy food, is interesting. Patients' sense of control towards food and the anticipation of consequences associated with food ingestion may explain these results. According to McNamara et al. (2008), when women suffering from an ED experience a feeling of loss of control over food, i.e. when they believe they cannot control their intake and their weight gain and/or their binge-purge behaviors, they may experience adverse emotions. Thus, it is possible that BN women thought they could manage their consumption of low and medium energy food because binge eating episodes mainly involve high sugar and fat products (Beumont, 2002; Gendall et al., 1997; Natenshon, 2009; Rosen et al., 1986). Therefore, food with lower energy value could have been considered at lower risk for inappropriate eating behaviors and potential weight gain. As a result, in the current study, the fear reaction to high-energy food was found to be more robust than fear reactions to lower energy food categories for $\mathrm{BN}$ participants.

For AN participants, their fear reactions were observed above and beyond their anxiety state prior to the experiment: the higher fear reactions observed among AN participants compared to $\mathrm{HC}$ and $\mathrm{BN}$ remained significant for all food energy density categories when controlling for participant's anxiety state. Therefore, the relationship between AN diagnosis and fear reactions to food appears to be robust. These results suggest that AN participants might experience a negative sense of control and anticipated the consequences of food intake for all caloric classes of food. This hypothesis relies on the idea that a core manifestation of $\mathrm{AN}$ is a morbid fear of gaining weight or becoming fat (APA, 2013). Then, even for low and medium energy food, women with AN could apprehend gaining some weight (or not maintaining their actual low weight) if they ingest them, as if all foods, independently of their caloric content, could represent a serious self-directed threat (Milos et al., 2017; Paslakis et al., 2016; Steinglass et al., 2007).

\section{Nutritional knowledge and its impact}

The results of the ranking task revealed that $\mathrm{AN}$ and $\mathrm{BN}$ women did not have better nutritional knowledge than $\mathrm{HC}$ about the energy density value of everyday food products. The three groups showed similar results when ranking food according to their caloric value. Furthermore, the results revealed that participants' nutritional knowledge, as we measured it, did not have a significant influence on their emotional responses; no association was found between women's nutritional knowledge and emotional reactions to food, regardless of their diagnosis. Therefore, it appears that ED participants' aversive reactions towards foods were not explained by their objective knowledge of caloric content. Instead, their emotional reactions may be influenced by their implicit cognitions about food and their subjective perception of food items' energy content, which seems to be erroneous (Eiber et al., 2005; Gonzalez \& Vitousek, 2004; Provencher et al., 2009; Sunday et al., 1992; Vartanian et al., 2004).

These findings leaded to the rejection of our hypotheses regarding nutritional knowledge, but the latter were mainly based on experiments held in the 1980s, at which time food and nutrition information was not as omnipresent in the public area as it is today (i.e., Beumont et al., 1981; Laessle et al., 1988). Nowadays, nutritional information is more abundant, elaborate, and readily available to the general population. It is possible that such a new social context has contributed to reduce the gap between HC and ED's nutritional knowledge about foods' energy value (Breen \& Espelage 2004; Soh et al., 2009).

\section{Strengths and limitations}

To our knowledge, this study is the first to explore ED participants' emotional responses to food according to their diagnosis and to the caloric content of the food products presented, while considering their comorbid affective state. Other strengths of the present study rely on the statistical control for participants' appetite level and on the investigation of the impact of their nutritional knowledge on their emotional reactions to foods. Furthermore, our experiment used a collection of standardized food pictures, which allowed to reduce the potential biases associated with different visual parameters, increasing the internal validity of our experiment. It should be noted that the set of food pictures used in this experiment (and their affective ratings) represents one of the first databases conceived for the examination of food-induced emotions in North-American ED individuals, most of those already available having been developed in Europe (e.g., FoodCast research image database, Foroni et al., 2013; Full 4 Health Image Collection, Charbonnier al., 2016; Open Library of Affective Foods; Miccoli et al., 2014, 2016). Thus, our bank of culturally representative food pictures could contribute to the development of clinical advances for North-American ED populations.

Despite its strengths, the present experiment has some limitations that must be mentioned. First, the fact that ED women, especially AN participants, may not be classified as critically ill as revealed by their outpatient status and their mean BMI outside the underweight category (i.e., > $18.5 \mathrm{~kg} / \mathrm{m}^{2}$ ). However, even if their illness's severity can appear to be low according to theses clinical aspects, the $\mathrm{AN}$ and BN participants of our study suffered from clear and significant ED symptoms, as assessed by a structured interview (i.e., module $\mathrm{H}$ of the SCID-I/Pl) and a specialized questionnaire (i.e., EDE-Q 6.0). Second, the absence of nonneutral non-food images - i.e., of pictures that could have evoked low happiness/joy and high disgust or fear responses like the food ones 
did-represents another limitation. The inclusion of such stimuli could have certified even more the specificity of food pictures in the induction of aversive reactions in ED women. Nevertheless, the inclusion of object images partially contributed to demonstrate this point. Third, the ranking task of food items according to their energy density to measure participants' nutritional knowledge may have been too difficult. This could have lowered their performance and reduced the probability to find differences between groups. Indeed, the task involved ranking many food products and some of them had a fairly similar caloric content. Finally, the representativeness of food's energy density as a measure of participants' nutritional knowledge can be discussed, as this element focuses on only one aspect of this knowledge. Consequently, the use of another type of nutritional knowledge measure-e.g., a questionnaire as those elaborated by Klieman et al., 2016; Saarela et al., 2013; and Dickson-Spillmann et al., 2011-could have been more relevant given the purpose of its inclusion.

\section{Conclusion}

This study demonstrated that ED women experienced a high level of fear towards food pictures, which was neither due to their level of hungriness nor to their affective comorbid state. This fear response appeared to be specific to food, because no difference between participants' emotional responses was noticed for object pictures. Most importantly, the study takes the literature further in the field of ED by revealing clear distinctions in women's reactions according to their diagnosis and according to the caloric content of the food presented. Precisely, for all food products displayed, regardless of their energy density, AN participants showed a more intense reaction of fear than $\mathrm{BN}$ and $\mathrm{HC}$. However, only highcalorie items led to a higher fear response in $\mathrm{BN}$ participants than in $\mathrm{HC}$, irrespectively of their anxiety state prior to the experiment. These results underline the necessity for treatments to address explicitly ED women's fear towards common food items and meals in a way of ensuring a longterm reduction of their symptoms and their recovery. In this perspective, our results support the use of therapeutic techniques that have been shown to be valid for anxiety disorders (e.g., gradual exposure and response prevention) in order to increase the effectiveness of psychological treatment of AN and BN (Levinson et al., 2014; Reilly et al., 2017; Steinglass et al., 2011; Treasure et al., 2015; Webb et al., 2011). Finally, the study revealed that participant's aversive reactions towards food pictures were not caused by their objective nutritional knowledge about them, which suggests that implicit cognitions might rather modulate their emotional responses. In summary, the study highlighted the key role of food-induced fear in ED's symptomatology, particularly in AN, and stressed the necessity of implementing treatments that address it exhaustively in order to improve patients' eating behaviors.

\section{References}

1. Aguiar-Bloemer, A. C., \& Diez-Garcia, R. W. (2018) Influence of emotions evoked by life events on food choice. Eating and Weight Disorders, 23, 45-53. doi: 10.1007/s40519-017-0468-8

2. Aharoni, R., \& Hertz, M. M. (2012). Disgust sensitivity and anorexia nervosa. European Eating Disorder Review, 20, 106110. doi: 10.1002/erv.1124

3. American Psychiatric Association (APA). (2013). Diagnostic and Statistical Manual of Mental Disorders (5 $5^{\text {th }}$ Edition). Arlington, VA: Author.

4. Aspen, V., Weisman, H., Vannucci, A., Nafiz, N., Gredysa, D., Kass, A. E., Taylor, C. B. (2014). Psychiatric co-morbidity in women presenting across the continuum of disordered eating. Eating Behaviors, 15, 686-693. doi: 10.1016/j.eatbeh.2014.08.023

5. Beumont, P. J., Chambers, T. L., Rouse, L., \& Abraham, S. F. (1981). The diet composition and nutritional knowledge of patients with anorexia nervosa. Journal of Human Nutrition, 35(4), 265-273.

6. Beumont, P. J. V. (2002). Clinical presentation of anorexia nervosa and bulimia nervosa, in Eating Disorders and Obesity, eds. C. G. Fairburn \& K. D. Brownell (New York, NY: Guilford Press), 162-170.

7. Beck, A. T., Steer, R. A., \& Brown, G. K. (1996). Beck Depression Inventory-II: Manual. San Antonio, TX: The Psychological Corporation.

8. Blechert, J., Meule, A., Busch, N. A., \& Ohla, K. (2014). Foodpics: an image database for experimental research on eating and appetite. Frontiers in Psychology, 5, Article 617. doi: 10.3389/fpsyg.2014.00617

9. Boehm, I., Flohr, L., Steding, J., Holzapfel, L., Seitz, J., Roessner, V. \& Ehrlich, S. (2018). The trajectory of anhedonic and depressive symptoms in anorexia nervosa: A longitudinal and cross-sectional approach. European Eating Disorder Review, 26, 69-74. doi:10.1002/erv.2565

10. Bradley, M. M., \& Lang, P. J. (1994). Measuring emotion: The Self-Assessment Manikin and the Semantic Differential. Journal of Behavioral Therapy and Experimental Psychiatry, 1, 49-59. doi: 10.1016/0005-7916(94)90063-9

11. Breen, H. B. \& Espelage, D. L. (2004). Nutrition expertise in eating disorders. Eating and Weight Disorders, 9, 120-125. doi: 10.1007/BF03325055

12. Brooks, S., Prince, A., Stahl, D., Campbell, I. C., \& Treasure, J. (2011). A systematic review and meta-analysis of cognitive bias to food stimuli in patients with disordered eating behaviour. Clinical Psychology Review, 31, 37-51. doi: 10.1016/jécpr.2010.09.006

13. Charbonnier, L., van Meer, F., van der Laan, L. N., Viergever, M. A., \& Smeets, P. A. M. (2016). Standardized food images: A photographing protocol and image database. Appetite, 96, 166173. doi: 10.1016/j.appet.2015.08.041

14. Cisler, J. M., \& Koster, E. H. W. (2010). Mechanisms of attentional biases towards threat in the anxiety disorders: An integrative review. Clinical Psychology Review, 30, 203-216. doi: 10.1016/j.cpr.2009.11.003

15. Coelho, J. S., Wilson, S., Winslade, A., Thaler, L., Israel M., \& Steiger, H. (2014). Over-evaluation of thoughts about food: Differences across eating -disorder subtypes and a preliminary examination of treatment effects. International Journal of Eating Disorders, 47, 302-309. doi: 10.1002/eat.22207

16. Dai, Q., \& Feng, Z. (2011). Deficient interference inhibition for negative stimuli in depression: An event-related potential study. Clinical Neurophysiology, 122, 52-61.

17. Dakanalis, A., Clerici, M., Bartoli, F., Caslini, M., Crocamo, C., Riva, G., \& Carrá, G. (2017). Risk and maintenance factor for young women's DSM-5 eating disorders. Archives of Women's Mental Health, 20, 721-731. doi: 10.1007/s00737-017-0761-6

18. Davey, G. C. L., Buckland, G., Tantow, B. \& Dallos, R. (1998). Disgust and eating disorders. European Eating Disorders Review, 6, 201-211. doi: 10.1002/(SICI)1099-0968(199809)6:3.

19. Davey, G. C. L., \& Chapman, L. (2009). Disgust and eating disorder symptomatology in a non-clinical population: The role of trait anxiety and anxiety sensitivity. Clinical Psychology and Psychotherapy, 16, 268-275. doi: 10.1002/cpp.623

20. Deborde, A. S., Berthoz, S., Godart, N., Perdereau, F., Corcos, M., \& Jeammet, P. (2006). Étude des relations entre alexithymia et anhédonie chez des femmes présentant des troubles du comportement alimentaire et chez des témoins. L'Encéphale, 32, 83-91. doi: 10.1016/S0013-7006(06)76140-1

21. Dickson-Spillmann, M., Siegrist, M., \& Keller, C. (2011). Development and validation of a short, consumer-oriented 
nutrition knowledge questionnaire. Appetite, 56, 617-20. doi: 10.1016/j.appet.2011.01.034

22. Drobes, D. J., Miller, E. J., Hillman, C. H., Bradley, M. M., Cuthbert, B. N., \& Lang, P. J. (2001). Food deprivation and emotional reactions to food cues: Implications for eating disorders. Biological Psychology, 57, 153-177. doi: 10.1016/S0301-0511(01)00093-X

23. Eiber, R., Mirabel-Sarron, C., \& Urdapilleta, I. (2005). Les cognitions et leur évaluation dans les troubles des conduits alimentaires. L'Encéphale, 31, 643-652. doi: 10.1016/S00137006(05)82422-4

24. Eizenman, M., Yu, L. H., Grupp, L., Eizenman, E., Ellenbogen, M., Gemar, M., \& Levitan, R. D. (2003). A naturalistic visual scanning approach to assess selective attention in major depressive disorders. Psychiatry Research, 118, 117-128. doi: 10.1016/S0165-1781(03)00068-4

25. Fairburn, C. G., \& Beglin, S. J. (2008). Eating disorder examination questionnaire (6.0), in Cognitive Behavior Therapy and Eating Disorders, ed. C. G. Fairburn (New York, NY: Guilford Press), 309-313.

26. Fairburn, C. G., Cooper, Z., \& Shafran, R. (2003). Cognitive behaviour therapy for eating disorders: A "transdiagnostic" theory and treatment. Behaviour Research and Therapy, 41, 509528. doi: 10.1016/S0005-7967(02)00088-8

27. First, M. B., Spitzer, R. L., Gibbon, M., \& Williams, J. B. W. (2002). Structured Clinical Interview for DSM-IV-TR Axis I Disorders. Research version. Patient edition (SCID-I/P). New York: Biometrics Research, New York State Psychiatric Institute.

28. Foroni, F., Pergola, G., Argiris, G., Rumiati, R. I. (2013). The FoodCast research image database (FRIDa). Frontiers in Human Neuroscience, 7, Article 51. doi: 10.3389/fnhum.00051

29. Foroughi, N., Madden, S., Clarke, S., Kohn, M., Donnelly, B., Touyz, S., \& Hay, P. (2018). Do emotional responses to food images differ within different types of eating disorders? Australasian Psychiatry, 1-6. doi: 10.1177/1039856218789790

30. Friederich, H.-C., Kumari, V., Uher, R., Riga, M., Schmidt, U., Campbell, I. C., Herzog, W., \& Treasure, J. (2006). Differential motivational responses to food and pleasurable cues in anorexia and bulimia nervosa: A startle reflex paradigm. Psychological Medicine, 36, 1327-1335. doi: 10.1017/S0033291706008129

31. Gagnon, C., Laflamme, V., Bégin, C., \& Grondin, S. (2018). Temporal processing of joyful and disgusting food pictures by women with an eating disorder. Frontiers in Human Neuroscience, 12, article 129. doi: 10.3389/fnhum.2018.00129

32. Gauthier, J., \& Bouchard, S. (1993). Adaptation canadiennefrançaise de la forme révisée du State-Trait Anxiety Inventory de Spielberger. Revue canadienne des sciences du comportement, 25, 559-578. doi: 10.1037/h0078881

33. Gendall, K. A., Sullivan, P. E., Joyce, F. A., Cater, F. A., \& Bulik, C. M. (1997). The nutrient intake of women with bulimia nervosa. International Journal of Eating Disorders, 21, 115-127. doi: 10.1002/(SICI)1098-108X(199703)21:2.

34. Giel, K. E., Teufel, M., Friederich, H.-C., Hautzinger, M., Enck, P., \& Zipfel, S. (2011). Processing of pictorial food stimuli in patients with eating disorders - A systematic review. International Journal of Eating Disorders, 44, 105-117. doi: 10.1002/eat.20785

35. Godart, N., Radon, L., Curt, F., Duclos, J., Perdereau, F., Lang, F. Flament, M. F. (2015). Mood disorders in eating disorder patients: Prevalence and chronology of ONSET. Journal of Affective Disorders, 185, 115-122. doi: 10.1016/j.jad.2015.06.039
Gonzalez, V. M. M. \& Vitousek, K. M. (2004). Feared food in dieting and non-dieting young women: A preliminary validation of the Food Phobia Survey. Appetite, 43, 155-173. doi: 10.1016/j.appet.2004.03.006

37. Griffiths, J., \& Troop, N. A. (2006). Disgust and fear rating of eating disorder-relevant stimuli: Associations with dieting concerns and fat intake. Anxiety, Stress, and Coping, 19, 421433. doi: 10.1080/10615800601066771

38. Harvey, T., Troop, N. A., Treasure, J. L., \& Murphy, T. (2002). Fear, disgust, and abnormal eating attitudes: A preliminary study. International Journal of Eating Disorders, 32, 213-218. doi: 10.1002/eat.10069

39. Hay, P., \& Katsikitis, M. (2014). Emotional responses to images of food in adults with an eating disorder: A comparative study of healthy and clinical controls. Eating Behaviors, 15, 371-374. doi: 10.1016/j.eatbeh.2014.04.016

40. Health Canada. (2011). Eating well with Canada's Food Guide. Ottawa, Canada.

41. Herzog, M., Douglas, C. R., Kissileff, H. R., Brunstrom, J. M., \& Halmi, K. A. (2017). Food portion size area mediates energy effects on expected anxiety in anorexia nervosa. Appetite, 112, 17-22. doi: 10.1016/j.appet.2017.01.012

42. Hoefling, A., Likowski, K. U., Deutsch, R., Häfner, M., Seibt, B., Mühlberger, A., Strack, F. (2009). When hunger finds no fault with moldy corn: Food deprivation reduces food-related disgust. Emotion, 9, 50-58. doi: 10.1037/a0014449

43. Houben, K., Roefs, A., \& Jansen, A. (2010). Guilty pleasures. Implicit preferences for high calorie food in restrained eating. Appetite, 55, 18-24. doi: 10.1016/j.appet.2010.03.003

44. Kemps, E., \& Wilsdon, A. (2010). Preliminary evidence for a role for impulsivity in cognitive disinhibition in bulimia nervosa. Journal of Clinical and Experimental Neuropsychology, 32, 515521. doi: 10.1080/13803390903264122

45. Kissileff, H. R., Brunstrom, J. M., Tesser, R., Bellace, D., Berthod, S., Thornton, J. C., \& Halmi, K. (2016). Computerized measurement of anticipated anxiety from eating increasing portions of food in adolescents with and without anorexia nervosa: Pilot studies. Appetite, 97, 160-168. doi: 10.1016/j.appet.2015.11.026

46. Kliemann, N., Wardle, J., Johnson, F., Croker, H. (2016). Reliability and validity of a revised version of the General Nutrition Knowledge Questionnaire. European Journal of Clinical Nutrition, 70, 1174-1180. doi: 10.1038/ejen.2016.87

47. Labonté, M.-È., Cyr, A., Baril-Gravel, L., Royer, M. M., \& Lamarche, B. (2012). Validity and reproducibility of a webbases, self-administrated food frequency questionnaire. European Journal of Clinical Nutrition, 66, 166-173. doi: 10.1038/ejen.2011.163

48. Laessle, R. G., Schweiger, U., Daute-Herold, U., Schweiger, M., Fichter, M. M., \& Pirke, K. M. (1988). Nutritional knowledge in patients with eating disorders. International Journal of Eating Disorders, 7, 63-73. doi: 10.1002/1098-108X(198801)7:1.

49. Lang, P. J. (1980). Behavioral treatment and bio-behavioral assessment: Computer applications, in Technology of Mental Health Care Delivery Systems, eds. J. B. Sidowski, J. H. Johnson \& T. A. Williams (Norwood, NJ: Ablex), 119-1 37.

50. Lang, P. J., Bradley, M. M., \& Cuthbert, B. N. (2008). International affective picture system (IAPS): Affective ratings of pictures and instruction manual. Technical Report A-8. Gainesville, Fl: University of Florida.

51. Leehr, E.J., Schag, K., Brinkmann, A., Ehlis, A. C., Fallgatter, A. J., Zipfel, S., Giel, K. E., Dresler, T. (2016). Alleged Approach-Avoidance Conflict for Food Stimuli in Binge Eating 
Disorder. PLoS One, 11, e0152271. doi: 10.1371/journal.pone.0152271

52. Levinson, C. A., Rapp, J., \& Riley, E. N. (2014). Addressing the fear of fat: Extending imaginal exposure therapy for anxiety disorders to anorexia nervosa. Eating and Weight Disorders, 19, 521-524. doi: 10.1007/s40519-014-0115-6

53. Libkuman, T. M., Otani, H., Kern, R., Viger, S. G., \& Novak, N. (2007). Multidimensional normative ratings for the International Affective Picture System [Supplemental material]. Behavior Research Methods, 39, 326-334.

54. Lowe, M. R., Arigo, D., Butryn, M. L., Gilbert, J. R., \& Sarwer, D. (2016). Hedonic hunger prospectively predicts onset and maintenance of loss of control eating among college women. Health Psychology, 35, 238-244. doi: 10.1037/hea0000291

55. Luce, K. H., \& Crowther, J. H. (1999). The reliability of the Eating Disorder Examination - Self-report questionnaire version (EDE-Q). International Journal of Eating Disorders, 25, 349351. doi: 10.1002/(SICI)1098-108X(199904)25:33.3.CO;2-D

56. Macht, M. (2008). How emotions affect eating: A five-way model. Appetite, 50, 1-11. doi: 10.1016/j.appet.2007.07.002

57. Macht, M., \& Simons, G. (2000). Emotions and eating in everyday life. Appetite, 35, 65-71. doi: 10.1006/j.appe.2000.0325

58. Martins, Y., \& Pliner, P. (2005). Human food choices: An examination of the factors underlying acceptance/rejection of novel and familial animal and nonanimal foods. Appetite, 45, 214-224. doi: 10.1016/j.appet.2005.08.002

59. McNamara, C., Chur-Hansen, A., \& Hay, P. (2008). Emotional responses to food in adults with an eating disorder: A qualitative exploration. European Eating Disorders Review, 16, 115-123. doi: 10.1002/erv.810

60. Mellers, B., Fincher, K., Drummond, C., Bigony, M. (2013). Surprise: A belief or an emotion?, in Progress in Brain Research, eds. V. S. C. Pammi \& N. Srinivasan (Amsterdam, Netherlands: Elsevier), 3-19. doi: 10.1016/B978-0-444-62604-2.00001-0

61. Miccoli, L., Delgado, R., Guerra, P., Versace, F., RodríguezRuiz, S., Fernández-Santaella, C. (2016). Affective pictures and the Open Library of Affective Foods (OLAF): Tools to investigate emotions toward food in adults. PLOS ONE, 11, e0158991. doi: 10.1371/journal.pone.0158991

62. Miccoli, L., Delgado, R., Rodríguez-Ruiz, S., Guerra, P., GarcíaMármol, E., \&, Fernández-Santaella, C. (2014). Meet OLAF, a good friend of the IAPS! The Open Library of Affective Foods: A tool to investigate the emotional impact of food in adolescents. PLoS ONE, 9, e114515. doi: 10.1371/journal.pone.014515

63. Michel, C., Velasco, C., Fraemohs, P., \& Spence, C. (2015a). Studying the impact of plating on ratings of the food served in a naturalistic dining context. Appetite, 90, 45-50. doi: 10.1016/j.appet.2015.02.030

64. Michel, C., Woods, A. T., Neuhäuser, M., Landgraf, A., \& Spence, C. (2015b). Rotating plates: Online study demonstrates the importance of orientation in the plating of food. Food Quality and Preference, 44, 194-202. doi: 10.1016/j.foodqual.2015.04.015

65. Milos, G., Baur, V., Schumacher, S., Kuenzli, C., Schnyder, U., Mueller-Pfeiffer, C., \& Martin-Soelch, C. (2017). How fat will it make me? Estimation of weight gain in anorexia nervosa. Appetite, 114, 368-373. doi: 10.1016/j.appet.2017.04.002

66. Mond, J. M., Hay, P. J., Rodgers, B., Owen, C., \& Beumont, P. J. V. (2004a). Validity of the Eating Disorder Examination Questionnaire (EDE-Q) in screening for eating disorders in community samples. Behaviours Research and Therapy, 42, 551-567. doi: 10.1016/S0005-7967(03)00161-X
Mond, J. M., Hay, P. J., Rodgers, B., Owen, C., \& Beumont, P. J. V. (2004b). Temporal stability of the Eating Disorder Examination Questionnaire. International Journal of Eating Disorders, 36, 195-203. doi: 10.1002/eat.20017

68. Mulveen, R., \& Hepworth, J. (2006). An interpretative phenomenological analysis of participation in a pro-anorexia internet site and its relationship with disordered eating. Journal of Health Psychology, 11, 283-96. doi: 10.1177/1359105306061187

69. Murray, S. B., \& Strigo, I. A. (2018). Anorexia nervosa, neuroimaging research, and the contextual salience of food cues: The food approach-avoidance conundrum. International Journal of Eating Disorders, 00, 1-4. doi: 10.1002/eat.22883

70. Natenshon, A. H. (2009). Eating disorders and the Diagnostic and Statistical Manual of Mental Disorders, in Doing What Works: An Integrative System for the Treatment of Eating Disorders from Diagnosis to Recovery, ed. A. H. Nathenshon (Washington, DC: NASW Press), 71-93.

71. Okon-Singer, H. (2018). The role of attention bias to threat in anxiety: Mechanisms, modulators and open questions. Current Opinion in Behavioral Sciences, 19, 26-30. doi: 10.1016/j.cobeha.2017.09.008

72. Paslakis, G., Kühn, S., Schaubschläger, A., Schieber, K., Röder, K., Rauh, E., \& Erim, Y. (2016). Explicit and implicit approach vs. avoidance tendencies towards high vs. low calorie food cues in patients with anorexia nervosa and healthy controls. Appetite, 107, 171-179. doi: 10.1016/j.appet.2016.08.001

73. Peterson, C. B., Crosby, R. D., Wonderlich, S. A., Joiner, T., Crow, S. J., Mitchell, J. E., le Grange, D. (2007). Psychometric properties of the Eating Disorder Examination-Questionnaire: Factor structure and internal consistency. International Journal of Eating Disorders, 40, 386-389. doi: 10.1002/eat.20373

74. Piech, R. M., Pastorino, M. T., \& Zald, D. H. (2010). All I saw was the cake: Hunger effects on attentional capture by visual food cues. Appetite, 54, 579-582. doi: 10.1016/j.appet.2009.11.003

75. Pike, K. M., Loeb, K., \& Walsh, B. T. (1995). Binge eating and purging, in Handbook of assessment methods for eating behaviors and weight-related problems: Measures, theory, and research, ed. D. B. Allison (Thousand Oaks, CA: Sage Publications Inc.), 303-346.

76. Piqueras-Fiszman, B., \& Spence, C. (2014). Colour, pleasantness, and consumption behavior within a meal. Appetite, 75, 165-172. doi: 10.1016/j.appet.2014.01.004

77. Provencher, V., Polivy, J., \& Herman, P. (2009). Perceived healthiness of food. If it's healthy, you can eat more. Appetite, 52, 340-344. doi: 10.1016/j.appet.2008.11.005

78. Racine, S. E. (2018). Emotional ratings of high- and low-calorie food are differentially associated with cognitive restraint and dietary restriction. Appetite, 302-308. doi: 10.1016/j.appet.2017.11.104

79. Racine, S. E., Hebert, K. R., \& Benning, S. D. (2018). Emotional reactivity and appraisal of food in relation to eating disorder cognitions and behaviours: Evidence to support the motivational conflict hypothesis. European Eating Disorders Review, 26, 3 10. doi: $10.1002 /$ erv. 2567

80. Reilly, E. E., Anderson, L. M., Gorrell, S., Schaumberg, K., \& Anderson, D. A. (2017). Expanding exposure-based intervention for eating disorders. International Journal of Eating Disorders, 50, 1137-1141. doi: 10.1002/eat.22761

81. Reisenzein, R., Horstmann, G., Schützwohl, A. (2017). The cognitive-evolutionary model of surprise: A review of the evidence. Topics in Cognitive Science, September, 1-25. doi: 10.1111/tops. 12292 
82. Robert-McComb, J. J., Wilson-Barlow, L., \& Goodheart, K. L. (2012). An overview of eating disorders, in Eating disorders in women and children: Prevention, stress management, and treatment, eds. K. L. Goodheart KL, J. R. Clopton \& J. J. RobertMcComb (Boca Raton, FL: CRC Press), 3-27.

83. Rodgers, R. F., Skowron, S., \& Chabrol, H. (2012). Disordered eating and group membership among members of a pro-anorexic online community. European Eating Disorders Review, 20, 912. doi: 10.1002/erv.1096

84. Rodríguez, S., Mata, J. L., Lameiras, M., Fernández, M. C., \& Vila, J. (2007). Dyscontrol evoked by erotic and food images in women with bulimia nervosa. European Eating Disorders Review, 15, 231-239. doi: 10.1002/erv.724

85. Rolls, B., \& Barnett, R A. (2000). Volumetrics: Feel full on fewer calories. New York: Harper Collins Publishers.

86. Rosen, J. C., Leitenberg, J., Fisher, C., \& Khazam, C. (1986). Binge-eating episodes in bulimia nervosa: The amount and type of food consumed. International Journal of Eating Disorders, 5, 255-267. doi: 10.1002/1098-108X(198602)5:2.

87. Rouleau, C. R., \& von Ranson, K. M. (2011). Potential risks of pro-eating disorder websites. Clinical Psychology Review, 31, 525-531. doi: 10.1016/j.cpr.2010.12.005

88. Rowley, J., \& Spence, C. (2018). Does the visual composition of a dish influence the perception of portion size and hedonic preference? Appetite, 128, 79-86. doi: 10.1016/j.appet.2018.06.005

89. Saarela, A.-M., Lapveteläinen, A. T., Mykkänen, H. M., Kantanen, T. T., \& Rissanen, R. L. (2013). Real-life setting in data collection. The role of nutrition knowledge whilst selecting food products for weight management purposes in a supermarket environment. Appetite, 71, 196-208. doi: 10.1016/j.appet.2013.08.014

90. Santel, S., Baving, L., Krauel, K., Münte, T. F., \& Rotte, M. (2006). Hunger and satiety in anorexia nervosa: fMRI during cognitive processing of food pictures. Brain Research, 1114, 138-148. doi: 10.1016/j.brainres.2006.07.045

91. Segal, D. L., Hersen, M., \& Hasselt, V. B. (1994). Reliability of the Structured Clinical Interview for DSM-III-R: An evaluative review. Comprehensive Psychiatry, 35, 316-327. doi: 10.1016/0010-440X(94)90025-6

92. Soh, N. L.-W., Touyz, S. W., Dobbins, T. A., Surgenor, L. J., Clarke, S., Kohn, M. R., Walter, G. (2009). Nutrition knowledge in young women with eating disorders in Australia and Singapore: A pilot study. Australian and New Zealand Journal of Psychiatry, 43, 1178-1184. doi: 10.3109/00048670903279846

93. Spence, C., Okajima, K., Cheok, A. D., Petit, O., \& Michel, C. (2016). Eating with our eyes: From visual hunger to digital satiation. Brain and Cognition, 110, 53-63. doi: 10.1016/j.bandc.2015.08.006

94. Spielberger, C. D. (1983). Manual for the State-Trait Anxiety Inventory. Palo Alto, CA: Consulting Psychologists Press Inc.

95. Steinglass, J. E., Eisen, J. L., Attia, E., Mayer, L., \& Walsh, B. T. (2007). Is anorexia nervosa a delusional disorder? An assessment of eating beliefs in anorexia nervosa. Journal of Psychiatric Practice, 13, 65-71. doi: 10.1097/01.pra.0000265762.79753.88

96. Steinglass, J. E., Sysko, R., Glasofer, D., Albano, A. M., Simpson, H. B., \& Walsh, B. T. (2011). Rationale for the application of exposure and response prevention to the treatment of anorexia nervosa. International Journal of Eating Disorders, 44, 134-141. doi:10.1002/est.20784

97. Stockburger, J., Schmälzle, R., Flaisch, T., Bublatzky, F., \& Schupp, H. T. (2009). The impact of hunger on food cue processing: An event-related brain potential study. NeuroImage, 47, 1819-1829. doi: 10.1016/j.neuroimage.2009.04.071

98. Stoeckel, L. E., Cox, J. E., Cook, E. W. 3rd, \& Weller, R. E. (2007). Motivational state modulates the hedonic value of food images differently in men and women. Appetite, 48, 139-144. doi: 10.1016/j.appet.2006.07.079

99. Sunday, S. R., Einhorn, A., \& Halmi, K. A. (1992). Relationship of perceived macronutrient and caloric content to affective cognitions about food in eating-disordered, restrained, and unrestrained subjects. American Journal of Clinical Nutrition, 55, 362-371. doi: 10.1093/ajen/55.2.362

100. Swinbourne, J., Hunt, C., Abbott, M., Russell, J., St Claire, T., \& Touyz, S. (2012). The comorbidity between eating disorders and anxiety disorders: Prevalence in an eating disorder sample and anxiety disorder sample. Australian \& New Zealand Journal of Psychiatry, 46, 118-131. doi: 10.1177/0004867411432071

101. Tokley, M., \& Kemps, E. (2007). Preoccupation with detail contributes to poor abstraction in women with anorexia nervosa. Journal of Clinical and Experimental Neuropsychology, 29, 734741. doi: 10.1080/13825580600966607

102. Treasure, J., Cardi, V., Leppanen, J., \& Turton, R. (2015). New treatment approaches for severe and enduring eating disorders. Physiology \& Behavior, 152, 456-465. doi: 10.1016/j.phybeh.2015.06.007

103. Troop, N. A., Treasure, J. L., \& Serpell, L. (2002). A further exploration of disgust in eating disorders. European Eating Disorders Review, 10, 218-226. doi: 10.1002/erv.444

104. Uher, R., Treasure, J., Heining, M., Brammer, M. J., \& Campbell, I. C. (2006). Cerebral processing of food-related stimuli: Effects of fasting and gender. Behavioral Brain Research, 169, 111-119 doi: 10.1016/j.bbr.2005.12.008

105. van Strien, T., Cebolla, A., Etchemendy, E., GutiérrezMaldonado, J., Ferrer-García, M., Botella, C., \& Baños, R. (2013). Emotional eating and food intake after sadness and joy. Appetite, 66, 20-35. doi: 10.1016/j.appet.2013.02.016

106. Vartanian, L. R., Polivy, J., \& Herman, P. (2004). Implicit cognitions and eating disorders: Their application in research and treatment. Cognitive and Behavioral Practice, 11, 160-167. doi: 10.1016/S1077-7229(04)80027-0

107. Vitousek, K. M., \& Brown, K. E. (2015). Cognitive-behavioral theory of eating disorders, in The Wiley handbook of eating disorders, eds. L. Smolak \& M. P. Levine (Malden, MA: John Wiley \& Sons, Ltd.), 222-236.

108. Wang, Y. P., \& Gorenstein, C. (2013). Psychometric properties of the Beck Depression Inventory-II: A comprehensive review. Revista Brasileira de Psiquiatria, 35, 416-431. doi: 10.1590/1516-4446-2012-1048

109. Webb, C. M., Thuras, P., Peterson, C. B., Lampert, J., Miller, D., \& Crow, S. J. (2011). Eating-related anxiety in individuals with eating disorders. Eating and Weight Disorders, 16, e236-41. doi: $10.1007 / \mathrm{bf03327466}$

110. Williams, J. B. W., Gibbon, M., First, M. B., Spitzer, R. L., Davis, M., Borus, J. Wittchen, H.-U. (1992). The Structured Clinical Interview for DSM-III-R (SCID). II. Multisite test-retest reliability. Archives of General Psychiatry, 49, 630-636. doi: 10.1001/archpsyc.1992.01820080038006

111. Williamson, D. A., Muller, S. L., Reas, D. L., \& Thaw, J. M. (1999). Cognitive bias in eating disorders: Implications for theory and treatment. Behavior Modification, 23, 556-577. doi: $10.1177 / 014544559923400$. 